\title{
Analysis of Diabetic Retinopathy by Detecting Exudates in the Retinal image
}

\author{
Gargi Kashyap \\ Asst. Professor \\ Dept. of ECE \\ GIMT, Guwahati
}

\begin{abstract}
Retinal image investigation and processing has great significance in clinical study. Digital retinal photograph is widely used for screening of the patients suffering from diseases such as Diabetic Retinopathy and Glaucoma. A common issue of diabetes is diabetic eye disease. Diabetes is the most common disease in India. Timely diagnosis and management of these diseases can prevent severe visual loss. The proposed work will mainly deal with the detection of clinical components exudates from the retinal images, which are of prime importance in the clinical examination of diabetic patients for automated diagnosis of retinal diseases. The proposed method has been chosen in order to reduce the time consumption, cost for detection of such diseases and to provide an easy, simple and efficient way. The Sensitivity and Specificity of the proposed work for analysis of disease in the retinal images of the patients suffering from DR from local hospital (Sankaradeva Nethralaya) are $96.9 \%$ and $98.4 \%$ and for STARE database are $95 \%$ and $97.5 \%$ respectively.
\end{abstract}

\section{General Terms}

Eye, Retinal Image, Diabetic Retinopathy, Fundas Image

\section{Keywords}

Diabetic Retinopathy (DR), Exudates, Macula, Mathematical Morphology operation (MM), Logical operation

\section{INTRODUCTION}

The human eye is the most complex organ in our body. The eye acts as a camera. The cornea behaving like a lens cover, the iris, pupil act like the aperture of a camera and the retina the inside back layer of the eye acting like a film. The retina is a membrane containing photoreceptor nerve cells that lines the inside back wall of the eye. The photoreceptor nerve cells of the retina change the light rays into electrical impulses and send them through the optic nerve to the brain where an image is perceived. The centre of the retina is called the macula. This is responsible for the sharp vision or reading vision. The peripheral retina is responsible for the peripheral vision. The human eye is remarkable [1].

Nowadays, diabetic retinopathy, glaucoma, and macular degeneration are the most common disease which causes visual impairment and blindness [2], [3].Early diagnosis and analysis of these diseases can prevent visual loss. Through regular ophthalmologic examination all of these diseases can

be detected but due to increase number of patients, the number of ophthalmologists needed for evaluation by direct examination is a limiting factor. So, an automatic system is required to detect the defeats in the retinal image so that it helps in diagnosing the disease.

Exudates are the primary factor if diabetic retinopathy which are mainly cause blindness. Exudates are yellowish or whitish patches of varying sizes, shapes. Diabetic Retinopathy is a type of disease which causes change in the blood vessels of the retina and as a result blood clotting happens near the macula and forms the exudates. It is one of the earliest and most prevalent symptoms of diseases leading to blindness such as diabetic retinopathy, age related macular degeneration. So detection of exudates is a very important.

There are two different types of Diabetic retinopathy namely Proliferative diabetic retinopathy (PDR) and Non Proliferative diabetic retinopathy (NDPR). In PDR, the blood vessel in the retina prevents the flow of blood in the eye. As a result it leads to formation of new blood vessels in order to supply blood to the blocked area. In NPDR, the impaired blood vessels leak extra fluid and as a result a small amount of blood comes into the eye. This condition leads to the formation of exudates in the retina near the macula. As the disease advances, the amount of exudates also increases in the retina. Figure 1 shows the normal retinal image and Figure 2 shows the retinal image with exudates.

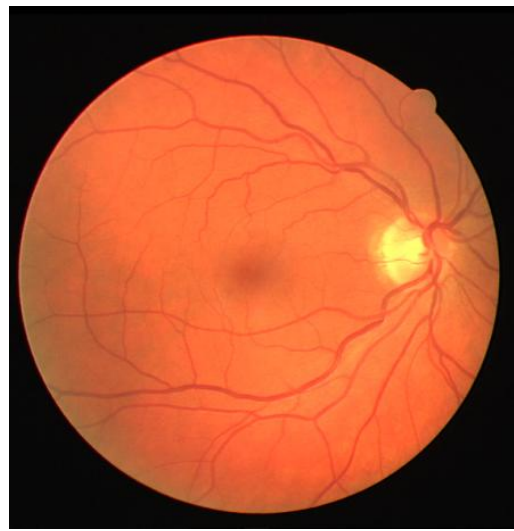

Figure 1: Normal Retinal Image

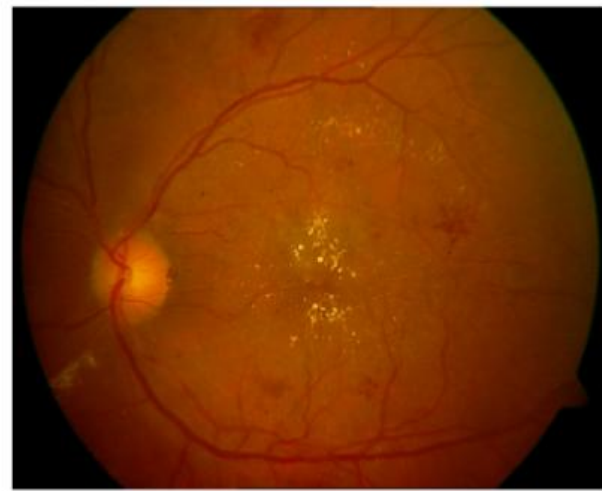

Figure 2: Retinal image with exudates 
The rest of the paper is organized as follows. In Section II, the literature survey is discussed. In Section III, the techniques used in the proposed algorithm are explained. In Section IV, experimental results of the algorithm are discussed. Finally, the conclusion of the paper is presented in Section V.

\section{LITERATURE SURVEY}

There are many approaches proposed in the literature for the detection of exudates $[2,3,4,5,6,7,8,9,10,11]$. Some of them are discussed below.

T.Yamuna, S.Maheswari presents a new approach to detect abnormalities in the retinal images in this paper [2]. To detect the abnormality, two preprocessing and one candidate extraction method are proposed and various stages of abnormalities are classified based on the features like area, mean standard deviation, entropy etc. Adaptive Neuro Fuzzy Inference System is used to classify the retinal images as normal, mild, severe depending on their severity.

In paper [3], a novel approach is made to detect macula in human retinal imagery. This paper demonstrates an approach to the rapid detection and extraction of macula from the images of human retina. For the detection, first bit plane decomposition is applied to the preprocessed image. The bit plane 0 and the bit plane 1 are found to carry vital information of the location and boundary of macula. Then the exact boundary by means of mathematical morphology is located. The algorithm is superior to the existing algorithms in terms of computational time and accuracy. A novel method for detection of exudates using Back Propagation Neural Method is proposed in this paper [4].This approach has sensitivity of $96.97 \%$, specificity of $100 \%$ and classification accuracy of $98.45 \%$.

In the paper [5], a process and knowledge of digital image processing to diagnose exudates from images of retina is applied. An automated method to detect and localize the presence of exudates and Maculopathy from low-contrast digital images of Retinopathy patient's with non-dilated pupils is proposed. First, the image is segmented using colour $\mathrm{K}$-means clustering algorithm. The segmented image along with optic disc is chosen .To classify the segmented region, features based on colour and textures are extracted. The selected feature vectors are then classified into exudates and non-exudates using a Support Vector Machine Classifier. Also the detection of Diabetic Maculopathy which is the severe stage of Diabetic Retinopathy is performed using Morphological Operation. Images with exudates were detected with $96 \%$ success rate. It can also detect very small areas of exudates. The presented work in paper [6] investigates and presents a morphology based techniques for the detection of diabetic retinopathy through exudates from color fundus images which includes the elimination of optic disc and the detected exudates are classified using image processing methods.

In this paper [7], an algorithm for exudates detection which is based on the combination of active contours obtained for the image after different preprocessing algorithms. The candidate regions of exudates with grayscale morphology are minimized by the Chan-Vese energy function to achieve the nearly accomplished boundary on nine different pre-processed images. In this paper [8], it focuses on Multi Layer Perception Neural Network (MLPNN) to detect diabetic retinopathy in retinal images. A feature vector is formed with 64-point Discrete Cosine Transform (DCT) with different statistical parameters.
The paper [9] presents an automatic method for exudates detection from colour fundus images based on Differential Morphological Profile (DMP). The image obtained from DMP contains highlighted bright regions consisting of exudates and optic disc. In the next phase, feature extraction based on location of optic disc, shape index and area is done to obtain actual exudates. The performance of the proposed method is evaluated by applying it on the DIARETDB1 database. The specificity, sensitivity and PPV of the proposed method were compared with two other methods. The results show that the proposed method gives better results than the other conventional methods. In the paper [10], the latest techniques in digital image processing and pattern classification are employed for detection of diabetic retinopathy. Various parameters like sensitivity, specificity, accuracy are used. The paper [11], proposed a computer assisted diagnosis based on the digital processing of retinal images for analysis of diabetic retinopathy. The pre processing is done and extraction of feature in done by Support vector machine to figure out the retinopathy grade of each retinal image. Parameters like sensitivity and predictive capacity are obtained.

\section{METHOD AND MATERIAL}

Detection of macula and exudates are important for the analysis of diabetic retinopathy disease. Under this condition, it shows considerable changes in the structure of macula and present of exudates may results in loss of vision in the patient. The proposed work demonstrates an approach to the rapid detection of exudates for the analysis of Diabetic Retinopathy, with the help of MATLAB.

\subsubsection{Image Acquistion}

The available database (STARE) and retinal images from the local eye hospital (Sankaradeva Nethralaya) are used for the proposed work.

\subsubsection{Pre Processing}

The retinal images from the local eye hospital (Sankaradeva Nethralaya), STARE database are used for giving as input to the system as shown in figure 3(a). The retinal image may have non-uniform illumination and varying intensity. So to overcome the problems, pre-processing is performed on the intensity component of an image. In this process of detection exudates, various color models like RGB, YCBCR and HSB models are implemented upon the retinal image separately. Among these models RGB model gives a clear view of the exudates. So, the RGB model is selected. In RGB model only the green channel as shown in figure $3(\mathrm{~b})$ is found to exhibit a better contrast level .So green channel is selected whereas the blue and red channels are avoided. Now the green plane image is contrasted and enhanced to make the components more visible and clear as shown in figure 3 (c). The green plane image is converted into gray scale as in the figure 3(d).

\subsubsection{Detection of Exudates}

In gray scale image, histogram equalization is done to get a uniform contrast retinal image. After that bit plane slicing is done for analyzing the relative importance of each bit of the retinal image as shown in figure 3(e), where the highest bit contains more number of information and the lowest bit contains the lowest number of information. Then logical operations are performed over the bit sliced retinal image. In the proposed method, logical XOR operation is performed as shown in figure 3(f). Then over the logical retinal image morphological operation are performed in which following operations are done. The operations are:

1. Opening: to remove noise and defects in the images. 
2. Closing: fills the small holes and gaps in a single pixel object.

Above operation are done by selecting a particular structural element which may be a square, disc, rectangle, hexagon, diamond etc. The selected structural element is square. After opening and closing image is complemented .The image is again opened, closed and then dilated to get the required exudates in the retinal image as shown in figure $3(\mathrm{~g})$.

The presence of Exudates indicates that the image taken is of person suffering from DR, otherwise image without exudates indicate a normal retinal image.

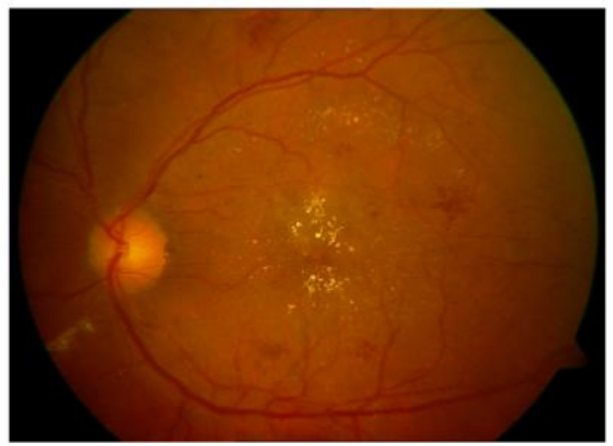

Figure 3 (a): Retinal Image with Exudates

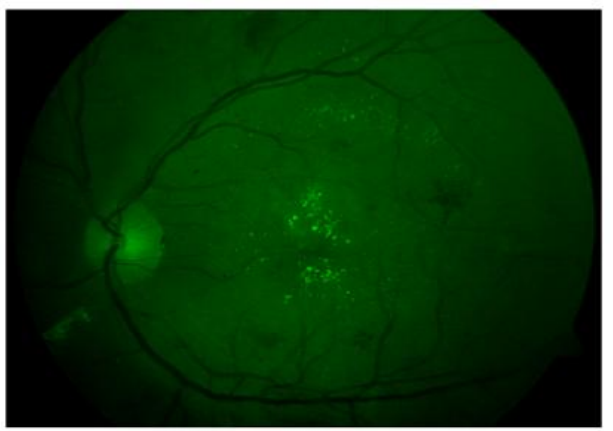

Figure 3 (b): Green Channel Retinal Image

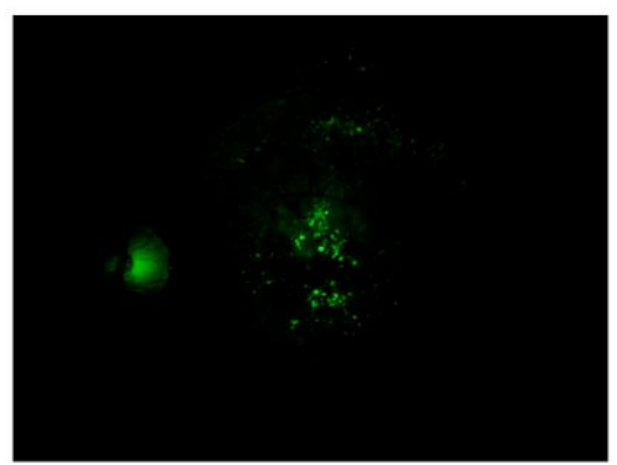

Figure 3(c): Contrasted Green Channel Image

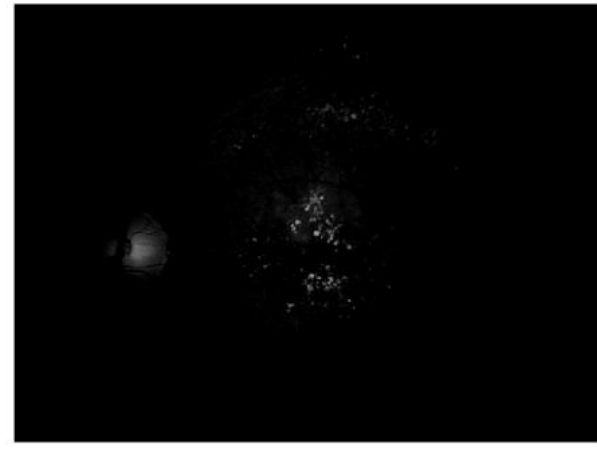

Figure 3(d): Gray Scale Retinal Image

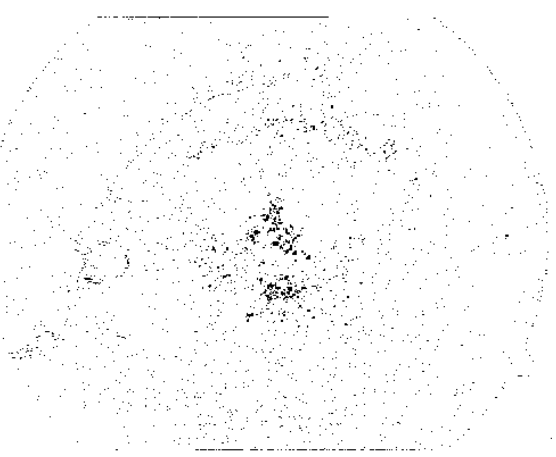

Figure 3(e): Bit plane sliced Retinal Image

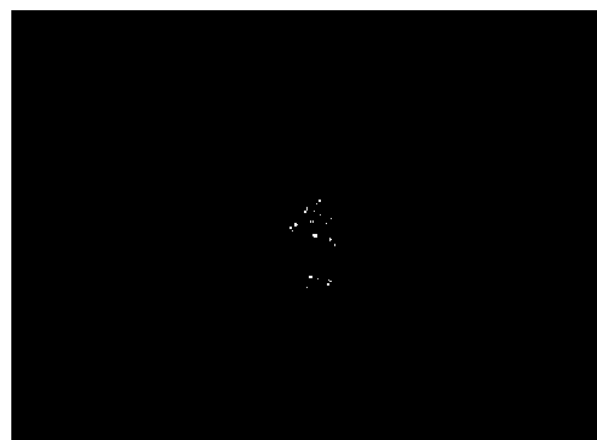

Figure 3(f): Logical operation Operated Image

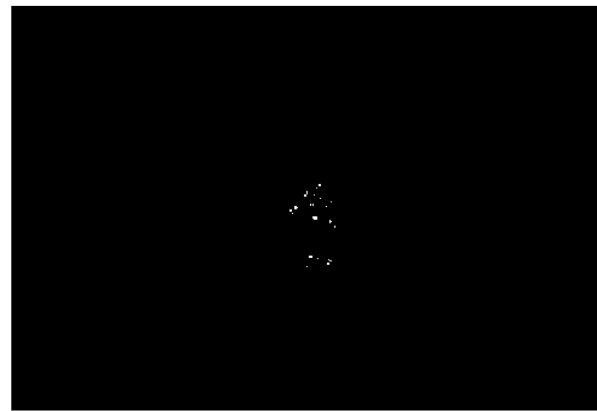

Figure 3(g): Exudates Detected in Retinal Image

\section{RESULT AND DISCUSSION}

In this section, the results of the proposed work are presented. Database of 105 images are used for evaluating the proposed work. The entire retinal images from various databases have sufficient variations in illumination and colour. The retinal image having exudates are considered as abnormal image and retinal image without exudates are normal image as classified by the ophthalmologists.

The retinal images are from various sources are as follows 65 
images from local Hospital (Sankaradeva Nethralaya) and 40 images from STARE databases. The performance is evaluated using the following parameters namely sensitivity and specificity. Sensitivity is defines as the percentage of actual exudates pixels that are detected and specificity is define as the percentage of non exudates pixels that are correctly classified as non exudates pixels [9], [10], [12].All these measures can be calculated based on four values: true positive (TP), the number of region of exudates correctly detected; false positive (FP), the number of region of non-exudates wrongly detected as exudates; false negative (FN), the number of region of exudates not detected and true negative (TN), the number of regions of non-exudates correctly identified. The qualitative result of exudates detection from various database based on the parameters are summarized in Table 1.

Table1. Results of exudates detection for specific database

\begin{tabular}{|l|l|l|l|}
\hline $\begin{array}{l}\text { Database } \\
\text { used }\end{array}$ & $\begin{array}{l}\text { Test } \\
\text { Image }\end{array}$ & Sensitivity & Specificity \\
\hline $\begin{array}{l}\text { Local } \\
\text { hospital }\end{array}$ & 65 & $96.9 \%$ & $98.4 \%$ \\
\hline STARE & 40 & $95 \%$ & $97.5 \%$ \\
\hline
\end{tabular}

\section{CONCLUSION}

In today's world every manual method are shifting to the automated computerised method for completion of any work of day to day life. By introducing new technologies, human labour has been minimised and the processing time is also reduced to great extent. The project is about the design of a system which will help in early detection of various retinal diseases such as age related macular degeneration, diabetic retinopathy etc. In the proposed work, fundus image of the retina taken with special fundus camera undergoes digital image processing to obtain the objective.

In normal process of detection of retinal diseases, the diagnosis becomes difficult and consumes much time due to lack of efficient methods. So a system is designed which will be efficient, cheap, less time consuming, simple and will help in rapid detection of the retinal diseases. The Sensitivity and Specificity of the proposed work for analysis of disease in the retinal images of the patients suffering from DR from local hospital (Sankaradeva Nethralaya) are $96.9 \%$ and $98.4 \%$ and for STARE database are $95 \%$ and $97.5 \%$ respectively.

The results confirm that the proposed method detects exudates quite efficiently and accurately. The segmentation of the exudates will be useful for ophthalmologists to diagnose diabetic retinopathy with a high degree of accuracy.

In the future to improve the accuracy of the proposed work, all the publicly available databases can be used to improve the accuracy and different parameters can be included to enhance the algorithm further.

\section{ACKNOWLEDGMENTS}

Lastly, we are greatly thankful to Sankaradeva Nethralaya for providing us their tested retinal images of diseased person which have helped us in the analysis of the DR disease.

\section{REFERENCES}

[1] Jayaraman. S., Esakkirajan. S., Veerakumar. T., "Digital Image Processing",Tata McGraw Hill, $5^{\text {th }}$ Edition, 2015.

[2] Yamuna.T.,Maheshwari.S., "Detection of Abnormalities in Retinal Images", IEEE International Conference on Emerging Trends in Computing, Communication and Nanotechnology(ICECCN), 2013.

[3] Murugan.R.,Korah.R., “An Automatic Screening Method to Detect Optic Disc in the Retina", International Journal of Advanced Information Technology, Vol.2, No.4, August 2012

[4] Karegowda. A.,Nasiha. A.,Jayaram. M. A., "Exudates Detection in Retinal Images Using Back Propagation Neural Network", International Journal of Advanced Computer Applications, Vol.25, No.3, July 2011.

[5] Ramasubramanian. B.,Mahendran. G., "An Efficient Integrated Approach for the Detection of Exudates and Diabetic Maculopathy in Colour Fundus Images", Advanced Computing: An International Journal, Vol.3, No.5, Sept 2012.

[6] Bachani.P., Patel. D., Parikh.R., "Automatic Detection of Exudates Using Morphological Approach ",IOSR Journal of Electronics and Communication Engineering, Vol.9,Issue1,Ver.1,2014.

[7] Harangi, B., Hajdu. A., "Improving Automatic Exudate Detection Based on the Fusion of the results of multiple Active Contours", IEEE $10^{\text {th }}$ International Symposium on Biomedical Imaging, 2013

[8] Bhatkar. P. A., Kharat. U. G., "Detection of Diabetic Retinopathy in Retinal Images Using MLP Classifier", IEEE International Symposium on Nanoelectronic and Information Systems, 2015.

[9] Tripathi. S., Singh. K. K., Singh. K.B., Mehrotra. A., "Automatic Detection of Exudates in Retinal Fundus Images using Differential Morphological Profile", International Journal of Engineering and Technology (IJET), Vol 5 No 3 Jun-Jul 2013.

[10] Ahmad. A., Mansoor. B.A., Mumtaz. R., Khan. M.,Mirza.H.S., "Image Processing and classification in diabetic retinopathy: A Review ", $5^{\text {th }}$ European Workshop on Visual Information Processing (EUVIP), 2014, DOI 10.1109/EUVIP.2014.7018362.

[11] Carrera.V. E., Gonzalez. A., Carrera. R., "Automated Detection of Diabetic retinopathy using SVM",IEEE XXIV International Conference on Electronics, Electrical and Computing (INTERCON), 2017, DOI: 10.1109/ INTERCON.2017.8079692

[12] Fraz. M. M., Basit. A. and Barman. A.S., "Application of morphological bit planes in retinal blood vessel extraction", Journal of Digital Imaging, Vol.26, No 2, pp274-286, April 2013. 